\title{
Positioning issues of spinal surgery during pregnancy
}

Daniele Bongetta ${ }^{1}$ AlessandroVersace ${ }^{2}$ Antonella De Pirro ${ }^{3}$ Marco Gemma ${ }^{4}$ Luca Bernardo ${ }^{5}$ Irene Cetin $^{6}$ Valeria Savasi ${ }^{7}$ Roberto Assietti ${ }^{2}$

1 Neurosurgery Unit, Fatebenefratelli e Oftalmico Hospital, Milan, Italy. Electronic address: danielebongetta@hotmail.com.

2 Neurosurgery Unit, Fatebenefratelli e Oftalmico Hospital, Milan, Italy.

3 Anesthesia and Intensive Care, University of Pavia, Pavia, Italy.

4 Anesthesia and Intensive Care Unit, Fatebenefratelli e Oftalmico Hospital, Milan, Italy.

5 Pediatrics Unit, Fatebenefratelli e Oftalmico Hospital, Milan, Italy.

6 Obstetrics and Gynecology Unit, "Ospedale dei bambini Vittore Buzzi" and University of Milan, Milan, Italy.

7 Obstetrics and Gynaecology Unit, "Luigi Sacco" Hospital and University of Milan, Milan, Italy.

\section{ABSTRACT \\ Background}

Albeit rarely, different spinal pathologies may require surgical treatment during pregnancy. The management of such cases poses a series of challenges, starting with adequate body positioning.

\section{Objective}

To illustrate limits and indications of the different surgical positioning strategies for pregnant women undergoing spine surgery.

\section{Methods}

We performed a systematic review of literature about the described surgical positioning strategies used for spinal surgery during pregnancy, discussing advantages, indications, and limits. We also describe of a novel three-quarters prone positioning for dorsal pathology.

\section{Results}

The surgical strategy may vary according to several factors, such as the location and the nature of the underlying pathology, the stage of the pregnancy, and the clinical condition of mother and fetus. During the second trimester, the habitus begins to raise issues about both the abdominal and the aortocaval compressions. The third trimester implies neonatal and ethical challenges: both fetal monitoring and the possibility of urgently proceeding to delivery should be guaranteed. The prone position is feasible during the second trimester provided an adequate frame is supplied. The lateral or three-quarters prone positioning may offer the safest option in the last stages of pregnancy, whereas both supine and sitting positionings are anecdotal. 


\section{Conclusions}

Gestational age, surgical comfort and maternofetal safety should be balanced by a multidisciplinary team to tailor an adequate positioning plan for each individual case. The early third trimester is the more limiting period because of the womb hindrance favoring lateral or three-quarters positionings.

KEY WORDS: Angiolipoma, Lateral, Pregnancy, Positioning, Prone, Position, Spine

\section{Abbreviations and Acronyms:}

MRI: Magnetic resonance imaging

\section{INTRODUCTION}

Spinal disorders are quite common during pregnancy; it has been reported that roughly $50 \%$ of childbearing women experience some kind of spine-related pain. ${ }^{1}$ Ligamentum laxity, relative osteoporosis, and, most obviously, both body mass index and habitus-related postural changes certainly play a significant role in this regard. $\underline{2}, \underline{3}, \underline{4}$ Luckily, the event of a serious spinal pathology requiring a surgical intervention is very rare because conservative management is usually the gold standard. $\underline{5}, \underline{6}, \underline{7}$ Nevertheless, a nonnegligible percentage of patients may have the necessity to undergo a surgical spinal procedure for different pathologies including tumoral, vascular, traumatic, or degenerative diseases. $\underline{8}, \underline{9}, \underline{10}, \underline{11}$ Although it is a physiological phenomenon, pregnancy is related to a series of anesthesiological, surgical and even ethical issues that may imply relevant variations from standard spinal surgical procedures. The surgical strategy may indeed vary according to several factors, such as the nature and location of the underlying pathology, the stage of the pregnancy, and the clinical conditions of mother and fetus. In this paper, we will focus on positioning issues. No systematic review of the suggested positionings and related caveats during spinal surgery in pregnant women has been performed to date. Our aim is to comprehensively illustrate the positioning options in this particular subset of patients, highlighting their pros and cons. Moreover, we present an illustrative case of an innovative three-quarters prone positioning option for midthoracic lesions we have designed for a rare thoracic angiolipoma.

\section{MATERIALS AND METHODS}

In January 2020, we electronically searched PubMed/MEDLINE, EMBASE, and The Cochrane Library to retrieve articles dealing with the positioning issues of spinal surgery during pregnancy, with no time or language restrictions. We used this search strategy in PubMed: (((position) OR positioning) AND spine) 
AND pregnant. Two independent researchers conducted literature searches to assess the actual relevance of a given article. Consensus was reached through discussion with the senior author if questions arose. References were cross-checked to retrieve further articles of interest. Papers with no clear description of body positioning were excluded and larger datasets were preferred for more common positions. A flow diagram according to PRISMA guidelines about the selection of the discussed articles is reported on Figure 1. While accounting for terminological variability, we schematized the data into the following 5 positionings: prone, lateral, three-quarters prone, supine, and sitting (including semisitting).

Given the consent of the patient, we present a case of a child-bearing woman operated on for a spinal tumor at our Institution.

\section{RESULTS}

In reviewing the topical literature, we acknowledged that several variables must be taken into account by the multidisciplinary team involved in surgically positioning a child-bearing woman. First, the underlining pathology may dictate the urgency of the intervention. Specifically, considering their relative incidences in the normal population, only $45 \%$ of reports were about degenerative diseases (mostly cauda equine syndrome in lumbar disk herniation), whereas fractures accounted for $24 \%$. Hematomas, infectious diseases, and tumors were reported as surgical indication in 14\%, 10\%, and 7\%, respectively. Moreover, several issues may rise as the pregnancy progresses. This is reflected by the prevalence in the gestational age reported with $3 \%, 41 \%$, and $56 \%$ of reports for first, second, and third trimesters, respectively. In fact, during the first trimester, no particular issue about positioning is to be found in literature, whereas pharmacologically and radiologically teratogenic effects are the most fearsome. From the second trimester on, the spatial clutter of the womb progressively becomes more prominent, giving rise to several problems. First, there is the need to avoid excessive anterior pressure on the womb itself. Second, the gravitational pressure exerted by the pregnant uterus on the retroperitoneal vascular structures may give rise to an aortocaval compression, with systemic and fetal vascular impairment. $\frac{12}{\text { Later, from }}$ the late second trimester on, there is the need to monitor the fetus because a significant distress is reported in about $5 \%$ of procedures. ${ }^{13}$ Eventually, there is the need to potentially be able to perform a preterm delivery procedure, even in an emergency setting. In this regard, there are some relevant limitations: to significantly decrease the likelihood of respiratory distress syndrome, any delivery should be postponed at least to the 32nd week of pregnancy or even later, given an appropriate treatment plan. There are several reported successful cases of single-stage general anesthesia with previous delivery and subsequent surgical treatment, thus limiting both patient's discomfort and bypassing all the pharmacological and positioning issues. $\stackrel{14}{ }$ However, especially in the early third trimester, potential fetal distress should be balanced against the mother's potential emergency situation, such as paraplegia, which in itself may 
complicate pregnancy. $\frac{15}{}$ This is therefore the period in which surgical positioning poses the greatest challenges, because in rare cases it is not possible to postpone spinal surgery after the delivery whilst the womb clutter has become highly relevant for anesthesiological, obstetrical, and surgical reasons. Every positioning has its advantages and relative/absolute contraindications in dealing with these limitations (Table 1). We are now going to systematically review all the suggested positioning found in literature.

Table 1. Positional Advantages From the Late Second Trimester on

\section{Surgical Comfort Fetal Comfort Cervical Thoracolumbar}

$\begin{array}{ccccc}\text { Prone } & ++ & -/+ & ++ & ++ \\ \text { Lateral } & -/+ & ++ & -/+ & -/+ \\ \text { Three-quarters prone } & + & ++ & + & + \\ \text { Supine } & ++ & + & ++ & - \\ \text { Sitting } & -/+ & ++ & ++ & -\end{array}$

\section{Prone}

Prone positioning is the workhorse for spinal procedures because of its direct access to most of the pathology as well as the superb surgical comfort for both operators. Despite the obvious spatial limitations of the progressively larger womb, there are some reports of this positioning after the first trimester too, albeit mainly limited to the second one. $\underline{10} \underline{15}, \underline{16}, \underline{17}, \underline{18}, \underline{19}, \underline{20}, \underline{21}, \underline{22}, \underline{23}, \underline{24}$ On the contrary, very few papers report the use of prone positioning in the third trimester. $\underline{\underline{8}} \underline{25}, \underline{26}, \underline{27}$ To avoid excessive pressure on the womb, some authors advocate the use of a Toronto or Relton-Hall frame, both equipped with a 4-pad lateral support system, which allows a wide space for the belly. $\underline{ }^{10}, \underline{17}$ Theoretically, even the use of a knee-chest position on an Andrews-type table may leave the womb with more space, even though abdominal pressure may not significantly vary. $\underline{28}$

On physiological grounds, there is some evidence that a prolonged prone position may not be as detrimental as previously thought, even for patients with preeclampsia. Nevertheless, these evaluations were performed only for a limited period (5 minutes); no further information is available on the physiological variations of mother's and fetus' parameters with a more prolonged decubitus. $\underline{29}$ Other crucial limitations of this positioning are the severe hindrance to fetal monitoring and the impossibility to perform an emergency delivery.

\section{Lateral}


For women in late pregnancy, the lateral decubitus position has been by far the most employed position

for the surgical treatment of the lumbar disk herniation, as highlighted in reviews. $, \underline{5}, \underline{30}$ Its advantages are manifold (e.g., that both fetal monitoring and the possibility to emergently resort to a delivery procedure are greatly favored). Moreover, because the left-side decubitus helps avoid the aortocaval compression, the cardiac vagal activity is affected the least whereas the cardiac sympathetic activity is enhanced as little as possible. ${ }^{12}$ Nevertheless, the surgical comfort during a left decubitus may be limited, especially for left-sided lumbar disk procedures. In this regard, the use of an endoscope has been advocated. $\frac{31}{\text { Still, }}$ other authors have postulated that the chance of developing a hemodynamically significant compression on the inferior vena cava and aorta during a short procedure in the right lateral procedure may be overemphasized. $\underline{11,32}$ Another peculiar advantage of the lateral position is the possibility to treat fractures by an anterior corridor. Specifically, when dealing with a good bone structure, a solely anterior reduction and stabilization of thoracolumbar fractures can be performed safely with sufficient expertise. Moreover, instrumented stabilization could be performed with less radiation exposure compared with posterior accesses. In particular, there have been reports of both thoracoscopic and retroperitoneal approaches in pregnant women. $\underline{33}, \underline{34}, \underline{35}$ No particular limitation has been reported when a transthoracic access is to be carried out. On the contrary, when performing the retroperitoneal access, it must be considered that the height of the fundus by the second trimester passes the fifth vertebral body progressing to the xiphoid process and the twelfth dorsal vertebra, thus potentially limiting such procedures in the late stages of pregnancy.

\section{Three-Quarters Prone}

This position is currently indicated with different names in the clinical setting of posterior fossa or occipital lesions. The three-quarters prone, lateral oblique, semiprone, or park-bench positionings share the same concept of exposing the cervicothoracic region in an inclined fashion with a good compromise between direct access to the lesion and surgical comfort. $\underline{36}, \underline{37}$ Incidentally, they also share the absence of excessive pressure on the abdominal region with the pure lateral position. Although it shows some potential advantages, we did not find any report in literature specifically describing its use. We designed a variant of this positioning without using a head holder and we tested it in our case as described in the following section.

At first, we also evaluated a standard park-bench position but, in our experience, positioning both the head holder and the inferior arm support may sometimes be lengthy and cumbersome and hence is contraindicated in such cases. Given the rotational potential of the cervical spine, park-bench positioning may be indicated in upper cervical spine lesions, provided the upper limbs are positioned more caudally, so as to enhance comfort for both operators.

\section{Supine}


There are few reports of supine positioning of pregnant patients all limited to cervical pathology. $\underline{38}, \underline{39}, \underline{40}$ No particular specific caveats are to be reported for this positioning apart from the prophylactic wedging of the right hip to avoid potential aortocaval compression. Moreover, in case of need of X-ray scans, a lead apron could be easily placed on the mother without interfering with the surgical procedures.

\section{Sitting}

Albeit anecdotal, few reports of using the sitting position are present in literature. ${ }^{41} \underline{42}$ Both the complexity of the setup and the fearsome potential complication of air embolism may have limited its use even in nonpregnant patients. Nevertheless, this positioning may show some nonnegligible advantages for cervical and upper thoracic lesions. First of all, it allows a direct access to the lesion site, with surgical advantages in terms of cleanliness of the surgical field due to gravity. Second, it allows posterior instrumented procedures, if needed, as reported in recent case series. ${ }^{43}, \underline{44}$ Last, it provides an easy, unhindered access to the womb, should there be any need for emergency procedures.

\section{Illustrative Case}

A healthy, 33-year-old primigravida 32-week-pregnant woman was admitted with rapidly progressing dorsal pain, gait imbalance, and bilateral leg paresthesias (Nurick score IV). An urgent magnetic resonance imaging (MRI) scan revealed an epidural mass at the level of T5-T7 extending laterally in the intervertebral foramina (Figure 2). The lesion was hypointense in T1, hyperintense in T2 and in short tau inversion recovery sequences, with homogeneous reinforcement of the signal after contrast medium administration. Inside the lesion, there were flow-void-compatible alterations as from rich vascularization. A slight medullary hyperintensity in $\mathrm{T} 2$ at the level of $\mathrm{T} 7$ was reported as from initial myelopathy. The following day she underwent surgery in a left-side modified three-quarters prone position as described in Figure 3. The intervention was performed under continuous tocographic monitoring and electrophysiological monitoring of somatosensory evoked potential, motor-evoked potential, and free-run electromyelography. The anatomical identification of the dorsal level was performed by means of direct distance measures from bony landmarks (inion and C7 spinal process) based on the sagittal MRI reconstruction. A decompressive laminectomy of T5-T6-T7 was performed, revealing an extradural, lipomatous mass with abundant arterial vascularization that was isolated from the posterior dura and excised en bloc. The pathological report was of angiolipoma. A posterior fixation was deemed unnecessary because of the intrinsic stability of the mid-dorsal level. Operative time was 150 minutes, blood loss was estimated at $750 \mathrm{~mL}$, hence a double red blood unit transfusion was performed during the following 12 hours. Postoperative course was uneventful. The patient progressively reverted to normal gait during the following month, she complained of residual bilateral leg paresthesias. A 3-week follow-up MRI revealed a gross total resection of the mass with persistence of the spinal cord T2 
hyperintense signal at the T7 level. She then naturally delivered a normal-term, healthy child. This represents the 16th pregnancy-related case of angiolipoma reported in literature, and only the second patient operated on during her pregnancy itself. $\underline{45}$

\section{Positioning}

The patient is laid on a left-side decubitus (Figure 3). The head rests on cushions, on the same axis of the spine and gently flexed anteriorly to better expose the cervical region. The left limb is gently flexed, whereas the right is kept straight, resting on a cushion between the 2 . This position avoids excessive pressure on bony prominences and enhances the support plane of the lower part of the body. Moreover, the right iliac crest is available for an anterior support to be set, thus allowing the trunk to be tilted up to about $30^{\circ}$. Given the little rotational potential of the thoracic spine, a gentle tilting of the operating table could be used to enhance the total tilt up to $45^{\circ}$. A small, cylindrical cushion is placed just below the axilla to slightly elevate the thorax from the bed plane, and the head of the bed is elevated about $15^{\circ}$ to avoid excessive pressure on the left glenohumeral and acromioclavicular joints. After generous padding on the pressure points, both upper limbs lie on horizontal arm rests fixed in a slightly cranial fashion. This gives space for both an easy access to the womb for monitoring issues and for the assistant to take place in a standard contralateral fashion. We believed this posture would allow a surgical maneuvering as standard as possible, while preserving both optimal fetal and maternal comfort and easiness to resort to an emergency delivery procedure. The extra tilting gained, in association with an adequate microscopic maneuvering, allows a thorough inspection of the intracanalar space. In particular, this also allows the inspection of contralateral foramina, which is usually precluded in standard lateral positioning.

\section{DISCUSSION}

This systematic review of the literature outlined the absence of evidence-based algorithms or large case series about the topic of positioning strategies in spinal surgery during pregnancy. This is the most relevant limitation to the conclusions of this study. Nevertheless, it permitted to underline several aspects of the management of spinal pathology during pregnancy that should be carefully evaluated in the choice of a correct positioning. Specifically, we stressed the concept of the gestational age as fundamental in the positioning choice. As reported, modern obstetrical and gynecological techniques may allow safe, early deliveries but, as in our case, an emergency neurological deterioration setting may foster a prompt intervention without the possibility to wait for a sufficient fetal development. $\stackrel{46}{\text { In }}$ this regard, we believe that a multidisciplinary team consisting of fetal-maternal physicians, obstetrics, spinal surgeons, and anesthesiologists is strongly advised to tailor an adequate plan for each individual case.

The three-quarters prone variant we have designed and described in this paper allows an easy fetal monitoring and avoids any positional impairment to the maternofetal circulation with an acceptable 
compromise in terms of surgical comfort and versatility. The pure "lateral" positioning is probably advantageous for its easiness and straightforwardness. Nevertheless, we fathomed that, using the microscope, a further tilting of the patient's torso allowed a better visualization and surgical comfort. Further studies are needed in evaluating new, more versatile, and ergonomic magnification devices such as exoscopes, which are rapidly gaining favor among the neurosurgical community.

\section{CONCLUSIONS}

Gestational age, surgical comfort, and maternofetal safety should be balanced by a multidisciplinary team to tailor an adequate positioning plan for each individual case. The early third trimester is the more limiting period because of the womb hindrance favoring lateral or three-quarters positionings.

\section{CRediT authorship contribution statement}

Daniele Bongetta: Conceptualization, Methodology, Data curation, Writing - original draft. Alessandro Versace: Data curation, Writing - review \& editing. Antonella De Pirro: Data curation, Writing - review \& editing. Marco Gemma: Methodology, Writing - review \& editing. Luca Bernardo: Writing - review \& editing. Irene Cetin: Writing - review \& editing. Valeria Savasi: Writing - review \& editing. Roberto Assietti: Conceptualization, Methodology, Writing - review \& editing, Supervision. 


\section{FIGURES}

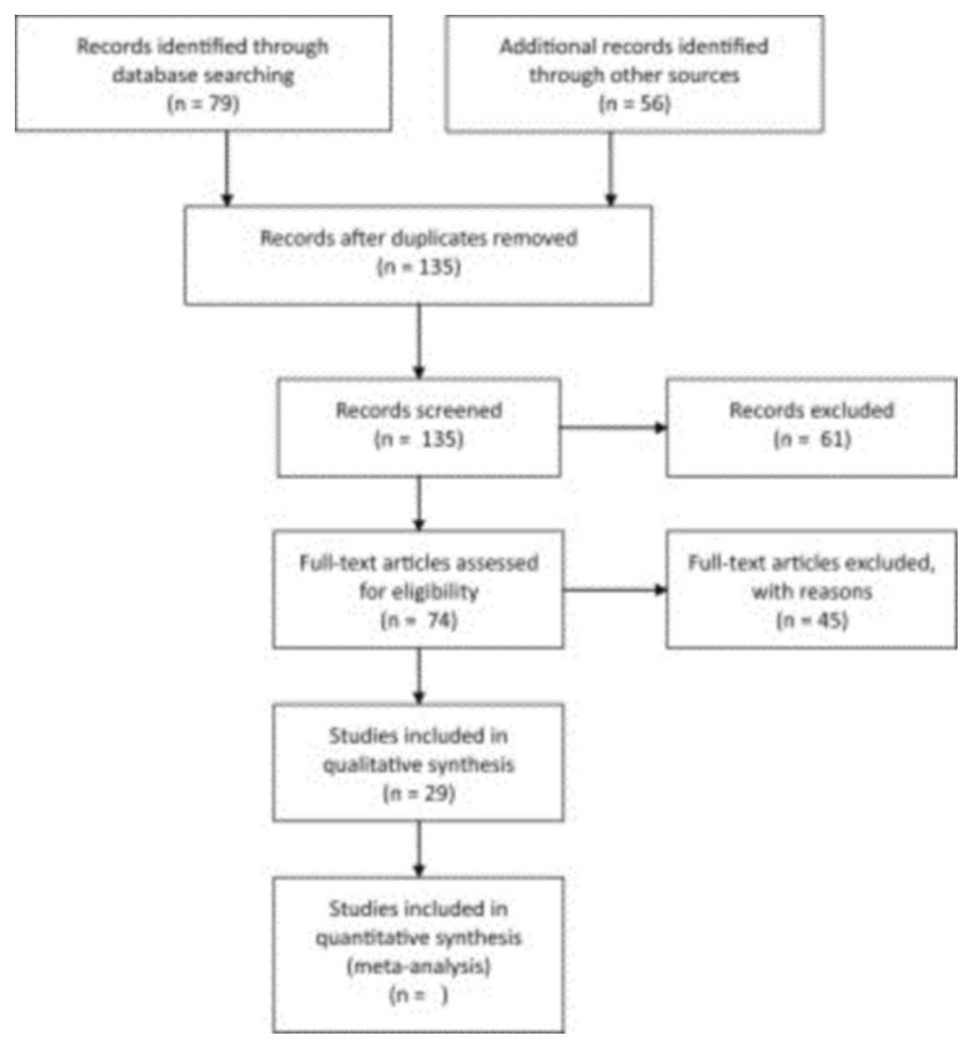

Figure 1. PRISMA Guidelines Flow Chart 


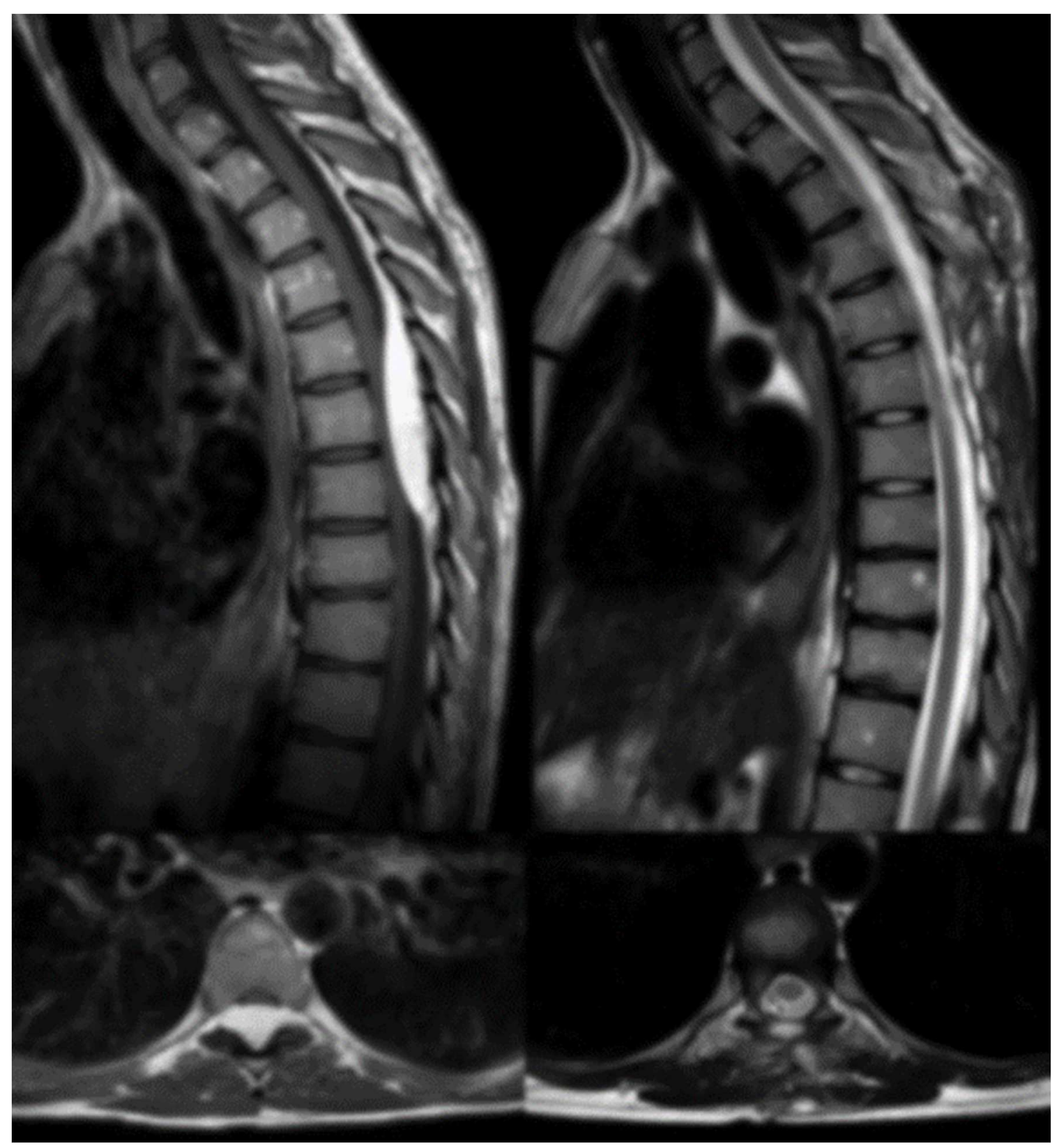

Figure 2. Sagittal and axial MRI reconstructions: (Left) Preoperative T1 contrast-enhanced sequences of spinal angiolipoma compressing the spinal cord and extending into the foramina bilaterally. (Right) Postoperative T2 sequences with a slight hyperintensity as for residual myelopathy. 


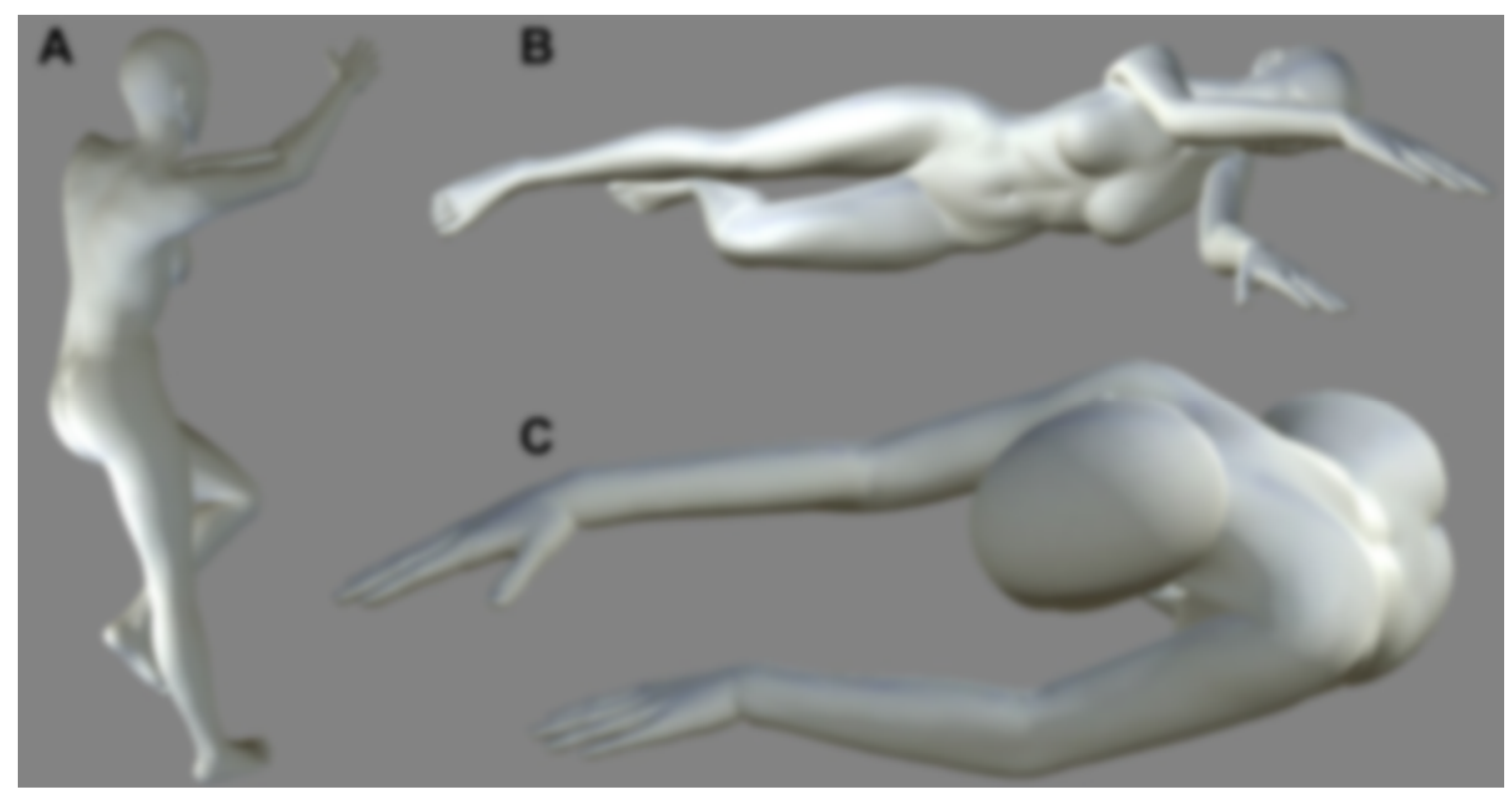

Figure 3. Three-dimensional rendering of the three-quarters prone positioning: (A) superior, (B) anterior, $(\mathbf{C})$ cranial views.

\section{SUPPLEMENTARY DATA}

\section{Data profile}

Conflict of interest statement: The authors declare that the article content was composed in the absence of any commercial or financial relationships that could be construed as a potential conflict of interest. 


\section{REFERENCES}

$1 \quad$ P. Katonis, A. Kampouroglou, A. Aggelopoulos, et al.Pregnancy-related low back pain Hippokratia, 15 (2011), pp. 205-210

$\underline{2}$ Y. Cherni, D. Desseauve, A. Decatoire, et al.Evaluation of ligament laxity during pregnancy J Gynecol Obstet Hum Reprod, 48 (2019), pp. 351-357

3 S.A. Hardcastle, F. Yahya, A.K. BhallaPregnancy-associated osteoporosis: a UK case series and literature review

Osteoporos Int, 30 (2019), pp. 939-948

$4 \quad$ J. Pauk, D. SwinarskaThe impact of body mass on spine alterations in pregnant women: a preliminary study

Technol Health Care, 26 (2018), pp. 665-669

$5 \quad$ A. Di Martino, F. Russo, L. Denaro, V. DenaroHow to treat lumbar disc herniation in pregnancy? A systematic review on current standards

Eur Spine J, 26 (Suppl 4) (2017), pp. 496-504

$\underline{6} \quad$ P. Kalinowski, A. KrawulskaKinesio taping vs. placebo in reducing pregnancy-related low back pain: a cross-over study

Med Sci Monit, 23 (2017), pp. 6114-6120

1 V.I. Vougioukas, G. Kyroussis, S. Gläsker, M. Tatagiba, K.M. ScheuflerNeurosurgical interventions during pregnancy and the puerperium: clinical considerations and management

Acta Neurochir (Wien), 146 (2004), pp. 1287-1291

discussion 1291-1292

$\underline{8} \quad$ T. Meng, H. Yin, Z. Li, et al.Therapeutic strategy and outcome of spine tumors in pregnancy: a report of 21 cases and literature review

Spine (Phila Pa 1976), 40 (2015), pp. E146-E153

$\underline{9}$ G. Levin, R. Haj-Yahya, R. Zigron, L. Matan, A. RottenstreichAn unusual case of spinal arteriovenous malformation presenting with severe paraplegia at term pregnancy Eur J Obstet Gynecol Reprod Biol, 225 (2018), pp. 268-269

10 D.V. Sonawane, P.S. Nemade, A. Chandanwale, E. Pawar, S.A. JagtapManagement of fracture dorso-lumbar spine in a pregnant female by surgery in prone position Eur Spine J, 27 (2018), pp. 270-275

11 V.Z. Kovari, L. HorvathSurgical management of cauda syndrome in third trimester of pregnancy focusing on spinal anesthesia and right lateral positioning during surgery as possible practices

Eur Spine J, 27 (2018), pp. 483-488 
12 C.D. Kuo, G.Y. Chen, M.J. Yang, Y.S. TsaiThe effect of position on autonomic nervous activity in late pregnancy

Anaesthesia, 52 (1997), pp. 1161-1165

13 G. Po', C. Olivieri, C.H. Rose, G. Saccone, R. McCurdy, V. BerghellaIntraoperative fetal heart monitoring for non-obstetric surgery: a systematic review

Eur J Obstet Gynecol Reprod Biol, 238 (2019), pp. 12-19

14 M.D. Brown, K.F. BrookfieldLumbar disc excision and cesarean delivery during the same anesthesia. A case report

J Bone Joint Surg Am, 86-A (2004), pp. 2030-2032

15 H. Göller, V. PaeslackPregnancy damage and birth-complications in the children of paraplegic women

Paraplegia, 10 (1972), pp. 213-217

16 K. Paonessa, R. FernandSpinal cord injury and pregnancy

Spine (Phila Pa 1976), 16 (1991), pp. 596-598

17 M.D. Brown, A.D. LeviSurgery for lumbar disc herniation during pregnancy

Spine (Phila Pa 1976), 26 (2001), pp. 440-443

18 K.M. Kuczkowski, S.A. Fouhsy, M. Greenberg, J.L. BenumofTrauma in pregnancy: anaesthetic management of the pregnant trauma victim with unstable cervical spine Anaesthesia, 58 (2003), p. 822

19 G.T. Chilkoti, M. Mohta, S. Duggal, A.K. SaxenaAnaesthetic concerns of a pregnant patient with Pott's spine for spine surgery in prone position

Indian J Anaesth, 60 (2016), pp. 518-519

20 M. Samali, A. Elkoundi, A. Tahri, M. Bensghir, C. HaimeurAnesthetic management of spontaneous cervical epidural hematoma during pregnancy: a case report J Med Case Rep, 11 (2017), p. 171

21 R. Vandse, M. Cook, S. BergeseCase report: perioperative management of a pregnant poly trauma patient for spine fixation surgery

Version 2. F1000Res, 4 (2015), p. 171

22 D.C.R. S, A.P. Shetty, R.M. Kanna, S. RajasekaranCauda equina syndrome in an obese pregnant patient secondary to double level lumbar disc herniation - a case report and review of literature

Spinal Cord Ser Cases, 5 (2019), p. 33

23 C.G. Martel, J. Volpi-Abadie, K. UralAnesthetic management of the parturient for lumbar disc surgery in the prone position

Ochsner J, 15 (2015), pp. 259-261 
24 T. HakanLumbar disk herniation presented with cauda equina syndrome in a pregnant woman

J Neurosci Rural Pract, 3 (2012), pp. 197-199

25 S. Tada, A. Yasue, H. Nishizawa, T. Sekiya, Y. Hirota, Y. UdagawaSpontaneous spinal epidural hematoma during pregnancy: three case reports

J Obstet Gynaecol Res, 37 (2011), pp. 1734-1738

26 S. Tsutsumi, Y. Nonaka, Y. Abe, Y. Yasumoto, M. ItoSpinal angiolipoma in a pregnant woman presenting with acute epidural hemorrhage

J Clin Neurosci, 18 (2011), pp. 849-851

27 U.M. Fahy, M. Oni, D. Findlay, P. SellSurgical management of herniated lumbar disc in pregnancy

J Obstet Gynaecol, 18 (1998), pp. 544-545

28 A. Rigamonti, M. Gemma, A. Rocca, M. Messina, E. Bignami, L. BerettaProne versus kneechest position for microdiscectomy: a prospective randomized study of intra-abdominal pressure and intraoperative bleeding

Spine (Phila Pa 1976), 30 (2005), pp. 1918-1923

29 A.T. Dennis, L. Hardy, L. LeetonThe prone position in healthy pregnant women and in women with preeclampsia - a pilot study

BMC Pregnancy Childbirth, 18 (2018), p. 445

$\underline{30}$ H. Ardaillon, Y. Laviv, J.E. Arle, E.M. KasperLumbar disk herniation during pregnancy: a review on general management and timing of surgery

Acta Neurochir (Wien), 160 (2018), pp. 1361-1370

31 K. Hayakawa, J. Mizutani, N. Suzuki, et al.Surgical management of the pregnant patient with lumbar disc herniation in the latter stage of the second trimester

Spine (Phila Pa 1976), 42 (2017), pp. E186-E189

32 I.H. Han, S.U. Kuh, J.H. Kim, et al.Clinical approach and surgical strategy for spinal diseases in pregnant women: a report of ten cases

Spine (Phila Pa 1976), 33 (2008), pp. E614-E619

33 K.J. Schnake, M. Scholz, A. Marx, R. Hoffmann, F. KandzioraAnterior, thoracoscopic-assisted reduction and stabilization of a thoracic burst fracture (T8) in a pregnant woman Eur Spine J, 20 (2011), pp. 1217-1221

34 R. Kaul, H.S. Chhabra, V. Kanagaraju, et al.Antepartum surgical management of Pott's paraplegia along with maintenance of pregnancy during second trimester Eur Spine J, 25 (2016), pp. 1064-1069

35 C.J. Lenarz, C.M. Wittgen, H.M. PlaceManagement of a pregnant patient with a burst fracture causing neurologic injury 
J Bone Jt Surg Am, 91 (2009), pp. 1747-1749

$\underline{6}$ G. Spena, F. Guerrini, G. GrimodA modified park bench position: the "Dormeuse" position Acta Neurochir (Wien), 161 (2019), pp. 1823-1827

37 V. Velho, H. Naik, A. Bhide, L. Bhople, P. GadeLateral semi-sitting position: a novel method of patient's head positioning in suboccipital retrosigmoid approaches Asian J Neurosurg, 14 (2019), pp. 82-86

$\underline{38}$ A.R. Pedaballe, H.S. Chhabra, V. Tandon, P. Chauhan, R. VermaAcute traumatic cervical spinal cord injury in a third-trimester pregnant female with good maternal and fetal outcome: a case report and literature review

Spinal Cord Ser Cases, 4 (2018), p. 93

39 S.A. Badve, S.D. Ghate, M.S. Badve, et al.Tuberculosis of spine with neurological deficit in advanced pregnancy: a report of three cases

Spine J, 11 (2011), pp. e9-e16

40 F. Feigenbaum, D.P. Sulmasy, E.D. Pellegrino, F.C. HendersonSpondyloptotic fracture of the cervical spine in a pregnant, anemic Jehovah's Witness: technical and ethical considerations. Case report

J Neurosurg, 87 (1997), pp. 458-463

41 D. Binnert, A. Thierry, R. Michiels, P. Soichot, M. Perrin[Presentation of a new case of spontaneous spinal extradural hematoma observed during labor]

J Med Lyon, 52 (1971), pp. 1307-1309

[in French]

42 Y. Yonekawa, H.M. Mehdorn, M. NishikawaSpontaneous spinal epidural hematoma during pregnancy

Surg Neurol, 3 (1975), pp. 327-328

43 C. Gan, J.A. King, N.F. MaartensThe role and safety of the sitting position in instrumented cervical surgery

J Clin Neurosci, 25 (2016), pp. 75-78

44 K. Leslie, R. Hui, A.H. KayeVenous air embolism and the sitting position: a case series J Clin Neurosci, 13 (2006), pp. 419-422

45 R. Maduri, A. Belouaer, J.P. Brouland, T. Ecker, M. MessererSpinal angiolipomas in pregnancy: natural history and surgical treatment

Clin Neurol Neurosurg, 178 (2019), pp. 25-30

46 S. Soltani, M.C. Nogaro, S.C. Jacqueline Kieser, M.C. Wyatt, D.C. KieserSpontaneous spinal epidural hematomas in pregnancy: a systematic review

World Neurosurg, 128 (2019), pp. 254-258 